\title{
Risk Assessment Method of Heavy Metal Pollution in Agricultural Soil
}

\author{
Shihong Huang ${ }^{1, a}$, Xianhao Long ${ }^{2, *}$, Guangxian Luo ${ }^{3}$, Lei Wang ${ }^{4}$ \\ ${ }^{1}$ Office of Science and Technology, Kunming University, Kunming, China \\ ${ }^{2}$ Development Planning Office, Kunming University, Kunming, China \\ ${ }^{3}$ Chuxiong Quality of the Technical Supervision and Inspection Center, Chuxiong, China \\ ${ }^{4}$ Chuxiong Notary Office of Yunnan Province, Chuxiong, China \\ a151688688@qq.com \\ *Corresponding author: longxh@kmu.edu.cn
}

Keywords: agricultural soil, heavy metal pollution, risk assessment.

\begin{abstract}
Heavy metal pollution of agricultural soil has regional and long-term characteristics. Scientific and reasonable pollution assessment is the premise of environmental pollution risk control and subsequent remediation strategy. This paper lists the advantages and disadvantages of common pollution risk assessment methods, and puts forward the recommended methods and suggestions.
\end{abstract}

\section{Introduction}

The heavy metal pollution of agricultural soil has regional and long-term characteristics. The heavy metal pollution of agricultural soil is closely related to the local industrial structure. The distribution of heavy metal pollution is closely related to the micro ecological division, soil type and planting structure. There are different characteristics of soil heavy metal pollution in different regions, and the risk of agricultural product safety and ecological security is also different. The classical data statistical analysis method lacks the consideration of spatial autocorrelation of soil heavy metal content, so there are great limitations in the research of key issues such as spatial distribution of soil heavy metal pollution, pollution source analysis and spatial statistical analysis of pollution. ${ }^{[1,2]}$ Therefore, scientific and reasonable evaluation of the pollution degree and distribution characteristics of heavy metals in the surrounding environment of agricultural soil is not only the theoretical basis and technical support of environmental quality and safety assessment, but also the premise of environmental pollution risk control and subsequent remediation strategy.

\section{Methods and problems of risk assessment of agricultural soil pollutants}

Pollutant risk assessment started from 1960 to 1970, which is a characteristic description of potential adverse effects on ecological environment or life and health due to hazard exposure in a period of time. Due to different assessment purposes and scales, the assessment indicators and methods of heavy metal pollution risk assessment in soil are also different. Although the total amount of heavy metals in soil can reflect the degree of soil pollution to a certain extent, its migration ability, biological toxicity and pollution risk to the environment cannot be described. ${ }^{[3]}$ The migration and biological toxicity of heavy metals in soil largely depend on their chemical forms and distribution. ${ }^{\text {[4] }}$

Academic institutions and scholars at home and abroad have put forward many assessment methods of soil heavy metal pollution ${ }^{[5]}$, including environmental standard quality method, single pollution index method, Nemerow pollution index method, geoaccumulation index method, enrichment factor method, secondary primary ratio method, fuzzy mathematics method, geostatistical assessment method based on GIS and human health risk assessment method ${ }^{[6]}$. Each evaluation method has advantages and disadvantages. At present, the scientific community has not put forward a widely recognized and generally accepted risk assessment method of heavy metal pollution in 
farmland soil. It is not enough to simply rely on the degree of soil heavy metal pollution or spatial distribution trend to identify and define the safety risks of heavy metal pollution to human health and ecological environment.

Table 1 Risk types of soil heavy metal pollution

\begin{tabular}{|c|c|}
\hline Risk category & Main pollution elements \\
\hline Human and animal health and food safety risk & $\mathrm{Cd}, \mathrm{Pb}, \mathrm{Hg}, \mathrm{As}$ \\
\hline Ecological environment security risk & $\mathrm{Ni}, \mathrm{Cu}, \mathrm{Zn}$ \\
\hline
\end{tabular}

The risk assessment of soil heavy metal pollution involves interdisciplinary, which is greatly restricted by methodology and detection means. The scale, purpose and method of heavy metal assessment vary with the types of heavy metals and the ways of soil utilization (such as farmland soil, industrial and mining sites, urban soil, garden soil, etc.). Specifically, soil heavy metals are mainly divided into two types according to risk types, as shown in Table 1.

In recent years, most of the risk assessment methods of heavy metal pollution in farmland only consider soil pollution, but ignore the response of soil to crop safety, and lack more scientific and practical assessment methods that comprehensively consider soil, plant and environmental factors. As a result, the domestic risk assessment methods of heavy metal pollution often have some problems, such as the use of evaluation standards are not unified, and the evaluation results are uncertain It also brings many problems for environmental pollution control and remediation. For example, in recent years, it has exposed many phenomena such as "soil exceeding standard, agricultural products not exceeding standard", "soil not exceeding standard, agricultural products exceeding standard", etc.

\section{Advantages and disadvantages of common pollutant risk assessment methods}

(1) Environmental standard quality evaluation method

It is easy to operate, but the evaluation standard is insufficient, so it is difficult to show the real level of soil pollution comprehensively and accurately.

(2) Pollution index evaluation method

Single pollution index method is simple and easy to operate, and it is difficult to show the degree of soil pollution comprehensively.

Nemero pollution index method - Nemero index method is one of the most commonly used methods to calculate comprehensive pollution index at home and abroad. In this method, the sub index (over standard multiple) of each factor is calculated first, and then the average value of each sub index is calculated, and the maximum sub index and average value are taken for calculation, which can fully show the harm degree of each heavy metal element to soil and highlight the influence of high concentration of heavy metal elements on soil quality. Because it is a comprehensive evaluation, it ignores the difference of the harmfulness of different heavy metals in the soil, and it is difficult to show the variability of heavy metal pollution characteristics; if there are abnormal values, the evaluation results will be distorted.

(3) Evaluation method of geoaccumulation index

Considering the effect of natural diagenesis on the background value, it can reflect the changes of regional soil characteristics and pollution sources, and directly show the heavy metal pollution level. Ignoring the correlation between heavy metal elements, the pollution ability and bioavailability of heavy metal elements, it is difficult to compare the environmental quality between elements and regions, and the constant $\mathrm{K}$ value selection is subjective.

(4) Enrichment factor evaluation method

The background value of heavy metal elements is considered, and the elements with less human pollution and stable chemical properties are taken as reference to reduce the error. The selection of reference elements is subjective.

(5) Secondary primary ratio evaluation method

The influence of heavy metal speciation was highlighted. Without considering the total amount of 
elements and background value, it is difficult to effectively compare the evaluation results with other evaluation methods, and some data are inconsistent with the pollution level.

(6) Fuzzy mathematics evaluation method

Considering the complexity of soil heavy metal pollution and the fuzziness of soil standard boundaries at all levels, the arbitrariness of determining the whole evaluation index is avoided through the optimal weight coefficient, and the evaluation results are true and reliable. Too much emphasis on the role of extreme value leads to incomplete information and too many membership functions lead to cumbersome process.

(7) Geostatistical evaluation method based on GIS

It can quickly obtain rich data, save manpower and material resources, identify pollution sources, intuitively display the distribution changes of regional pollution, reduce the subjectivity of the evaluation method, and improve the accuracy. There are some limitations, if the spatial correlation of regionalized variables does not exist, it is not applicable.

(8) Risk assessment method based on human health

By estimating the probability of heavy metal elements harming human health, the priority pollutants were determined, which provided the basis for environmental governance. This method only considers the impact on human health and ignores other impact objects. There are deficiencies and uncertainties in the evaluation process.

\section{Recommended risk assessment methods for agricultural soil pollutants}

On October 14th, 2015, the Ministry of Agriculture issued the technical regulations on the safety assessment of heavy metals in the soil of agricultural products producing areas (No.42 of the agricultural products office [2015], taking into account the factors such as agricultural products types, soil physical and chemical properties, and aiming at ensuring the quality and safety of edible agricultural products, the Ministry of agriculture formulated a double index risk assessment method for heavy metal pollution in farmland soil, including heavy metals of $\mathrm{Cd}, \mathrm{Hg}, \mathrm{Pb} \mathrm{Cr}_{5}$ in the soil. The method of soil plant double index evaluation is based on the single factor index, which is the scientific and reliable method of the comprehensive evaluation method established by the single factor index of soil and single factor index of agricultural products.

\section{Conclusions}

(1) Soil plant dual index evaluation method is composed of single factor index of soil heavy metals and single factor index of agricultural products. To a certain extent, it considers the impact of soil pollution level on the accumulation of heavy metals in edible parts of crops, reflects the plant availability of heavy metals in farmland soil under different utilization methods, and also reflects the impact of different types and varieties of crops on different heavy metals to a certain extent There are inherent differences in bioaccumulation. Soil plant dual index assessment method improves the accuracy and scientificity of farmland heavy metal pollution risk assessment to a certain extent, and has a substantial guiding role for environmental remediation and early warning. However, compared with soil environmental standard quality assessment method, it is more complex, and there are still some shortcomings, such as not considering the trend of pollution accumulation, unable to assess the potential risk of farmland soil. It is suggested that more consideration should be given to this influencing factor.

(2) In the evaluation, the sampling plots are generally located in agricultural areas, which are greatly affected by manual operation, field stacking, the concentration of crop distribution and the degree of heavy metal contact in the plots. Therefore, it should pay attention to the reasonable allocation of points in different areas. When determining the location and sampling depth of the survey and detection points, it should also judge the growth characteristics and speed of the roots of the crops. In order to get more accurate data, the method of encrypting points can be used for agricultural land with serious pollution. 


\section{Acknowledgments}

This work was financially supported by Yunnan Local Colleges Applied Basic Research Projects (No.2017FH001-053) and Yunnan Local Colleges Applied Basic Research Projects (No.2018FH001-054).

\section{References}

[1] S. Wang, MA J.J. Ma, D.Zhao, P.T. Lei, Pollution Characteristics and Potential Ecological Risk Assessment of Soil Metals in the North Area of Shaanxi Province, China, 2013, 30(5):44-47

[2] Y.H. Jia, J.X. Guo, J. Liu, J.X. Wang, J.D. Du, F. Gao. Vertical Distribution of Heavy Metals in Soil of Facility Vegetable Fields from Beijing Suburbs, China, 2015, 32(2):282-288.

[3] H.C. Cao, J.D. Wang, X.L. Zhang, Application of Sequential Extraction of BCR to Forms Distribution of Heavy Metals in Polluted Black Soil[J]. Journal of Soil and Water Conservation, 20(6):163-166.

[4] C. GLEYZES, S. TELLIER, M., ASTRUC. Fractionation studies of trace elements in contaminated soils and sediments:a review of sequential extraction procedures[J]. Tr AC Trends in Analytical Chemistry, 2002, 21(6/7):451-467.

[5] Z. Xiong, Studies on Pollution Characteristics and Risk Assessment of Heavy Metal in HeBei's Farmland Soil -A Case of Cadmium and Nickel[D]. Chinese Academy of Agricultural Sciences, 017.

[6] Y.J. wang, T.L. Wu, D.M. Zhou, H.M. Chen, Advances in soil heavy metal pollution evaluation based on bibliometrics analysis, Journal of Agro-Environment Science, 2017,36(12):2365-2378. 